\title{
Biomedical Informatics Training at the University of Wisconsin-Madison
}

\author{
D. J. Severtson ${ }^{1,2, L .}$ Pape', C. D. Page, Jr. ${ }^{1,4,6}$, J. W. Shavlik ${ }^{1,4,6}$, G. N. Phillips, Jr. ${ }^{1,5,6}$, P. Flatley \\ Brennan ${ }^{1,2,3,4}$ \\ ${ }^{1}$ Computation and Informatics in Biology and Medicine Program \\ ${ }^{2}$ School of Nursing \\ ${ }^{3}$ Department of Industrial and Systems Engineering \\ ${ }^{4}$ Department of Biostatistics \& Medical Informatics \\ ${ }^{5}$ Department of Biochemistry \\ ${ }^{6}$ Computer Sciences Department \\ All authors are from the University of Wisconsin-Madison, USA
}

\begin{abstract}
Summary
Objectives: The purpose of this paper is to describe biomedical informatics training at the University of Wisconsin-Madison (UWMadison).

Methods: We reviewed biomedical informatics training, research, and faculty/trainee participation at UW-Madison.

Results: There are three primary approaches to training 1) The Computation \& Informatics in Biology \& Medicine Training Program, 2) formal biomedical informatics offered by various campus departments, and 3) individualized programs. Training at UW-Madison embodies the features of effective biomedical informatics straining recommended by the American College of Medical Informatics that were delineated as: 1) curricula that integrate experiences among computational sciences and application domains, 2) individualized and interdisciplinary crosstraining among a diverse cadre of frainees to develop key competencies that he or she does not initially possess, 3) participation in research and development activities, and 4) exposure to a range of basic informational and computational sciences.

Conclusions: The three biomedical informatics training approaches immerse students in multidisciplinary training and education that is supported by faculty trainers who participate in collaborative research across departments. Training is provided across a range of disciplines and available at different training stages. Biomedical informatics training at UW-Madison illustrates how a large research University, with multiple departments across biological, computational and health fields, can provide effective and productive biomedical informatics training via multiple bioinformatics training approaches.
\end{abstract}

\section{Keywords}

Academic training, training programs, informatics, biomedical informatics

Geissbuhler A, Haux R, KulikowskiC, editors. IMIA Yearbook of Medical Informatic 2007. Methods Inf Med 2007; 46Supp 1: 149-56

\section{Introduction}

A plethora of approaches support biomedical informatics training at the University of Wisconsin-Madison, all of which share a strong focus on interdisciplinary coursework and research. There are three primary approaches to training 1) The Computation \& Informatics in Biology \& Medicine Training Program, 2) formal biomedical informatics offered by various campus departments, and 3) individualized programs. Training is supported by institutional and individual pre-doctoral, postdoctoral, and career fellowships. Biomedical informatics training at UWMadison embodies features that were outlined in a report from a study of the future of informatics training conducted by the American College of Medical Informatics [1]. This study committee defined biomedical informatics as an interdisciplinary field that combines basic informational and computational sciences with application domains that include health care, biological research, and education. They concluded that effective training in informatics consists of: 1) curricula that integrate experiences among computational sciences and application domains, 2) individualized and interdis- ciplinary cross-training among a diverse cadre of trainees to develop key competencies that he or she does not initially possess, 3) participation in research and development activities, and 4) exposure to a range of basic informational and computational sciences. The following description of biomedical informatics training at UW-Madison illustrates that these elements form the foundation for productive and integrated training across a spectrum of biomedical informatics application domains.

\section{Computation \& Informatics in Biology \& Medicine Training Program}

The Computation \& Informatics in Biology \& Medicine (CIBM) Training Program is a bioinformatics training program for pre-doctoral and postdoctoral trainees, funded by the National Library of Medicine (NLM) since 2002. CIBM's mission is to provide modern training for a new generation of researchers wishing to solve biomedical problems requiring strengths in both computational and biological science. 


\section{Faculty \& Trainees}

The 41 CIBM faculty span 15 different departments and five colleges at the University of Wisconsin-Madison, and include several at the Marshfield Clinic Research Foundation, as well. The research foci of CIBM faculty are available on the CIBM Program website at www.cibm.wisc.edu.The management team includes George Phillips (Professor of Biochemistry and of Computer Sciences) as the Program Director, David Page (Associate Professor of Biostatistics \& Medical Informatics and of Computer Sciences) and Jude Shavlik (Professor of Computer Sciences and of Biostatistics \& Medical Informatics) as Co-directors, and a Management Committee that consists of Frederick Blattner (Oliver Smithies Professor of Genetics), Patricia Flatley Brennan (Moehlman Bascom Professor of Nursing, Industrial \& Systems Engineering, and of Biostatistics \& Medical Informatics), Mark Craven (Associate Professor of Biostatistics \& Medical Informatics and of Computer Sciences), David DeMets (Professor and Chair of Biostatistics \& Medical Informatics and Professor of Statistics), and Justin Starren (Director of the Biomedical Informatics Research Center, Marshfield Clinic Research Foundation).

The departmental homes of CIBM trainers who have served as primary mentors for CIBM trainees and CIBM trainees are summarized in Table 1. The percentages of trainees and faculty trainers differ because many faculty have joint appointments, and the Biostatistics \& Medical Informatics Department does not directly accept graduate students. Joint appointments among faculty facilitate integrated training. Pre and postdoctoral trainees select a primary and a secondary mentor that reflect their primary and cross-training foci respectively. Several students have

\begin{tabular}{|c|c|c|c|}
\hline \multirow{13}{*}{$\begin{array}{l}\text { Affiliations of Primary } \\
\text { Faculty Trainers and Pre- } \\
\text { doctoral Trainees }\end{array}$} & Department & Trainers & Trainees \\
\hline & Computer Sciences & $20 \%$ & $51 \%$ \\
\hline & Biostatistics and Medical Informatics & $31 \%$ & - \\
\hline & Biochemistry & $17 \%$ & $14 \%$ \\
\hline & Chemical \& Biological Engineering & $9 \%$ & $9 \%$ \\
\hline & Biophysics Graduate Program & - & $6 \%$ \\
\hline & Industrial \& Systems Engineering & $3 \%$ & $3 \%$ \\
\hline & Mathematics & $6 \%$ & $3 \%$ \\
\hline & Biomedical Engineering & $6 \%$ & $6 \%$ \\
\hline & Chemistry & $3 \%$ & $3 \%$ \\
\hline & Genetics & $3 \%$ & - \\
\hline & School of Nursing & $3 \%$ & $3 \%$ \\
\hline & Special Bioinformatics PhD & - & $3 \%$ \\
\hline
\end{tabular}

constructed a special multidisciplinary bioinformatics $\mathrm{PhD}$ degree.

Additional CIBM faculty trainers are in the Departments of Electrical and Computer Engineering, Oncology, and Statistics. Many are members of the Genome Center of Wisconsin, which administers the CIBM Training Program. Two CIBM faculty are affiliated with the Marshfield Clinic. The Marshfield Clinic is located in 41 centers throughout northern, central and western Wisconsin. Its research division, the Marshfield Clinic Research Foundation, conducts research on healthcare and public health. Areas of focus include clinical research, rural and agricultural health and safety, human genetics, epidemiology and biomedical informatics.

\section{CIBM Curriculum}

The CIBM program is not a degree granting program but designed to foster interdisciplinary training. CIBM trainees become well versed in a common language of concepts from computer science, statistics, and biology.
Students can use the required CIBM classes to satisfy their PhD minor requirement in their respective home departments. The CIBM Program's curriculum for pre-doctoral trainees (see Table 2) has three components:

- Interdisciplinary bioinformatics core courses: All pre-doctoral students are required to take an introductory class in biostatistics, an essential skill in an increasingly "data driven" world, as well as Introduction to Bioinformatics. The third course in this group can be chosen from a set of advanced biomedical informatics courses.

- Molecular-biology training triad: Students choose three classes from a set of genetics, genomics, and biochemistry courses that best match their research goals to provide education in the basics of molecular biology.

- Basics of computer science: All students are expected to take Introduction to Data Structures. The other classes address different aspects of computation that play key research roles in bioinformatics and scientific computation in general.

All CIBM trainees participate in a 


\section{Table 2 CIBM Curriculum}

\begin{tabular}{|l|l|}
\multicolumn{2}{|c|}{ Bioinformatics / Biostatistics (3 required) } \\
\hline Stat 571 & Statistical Methods for Bioscience I \\
BMI 576 & Intro. to Bioinformatics \\
& \\
BMI 776 & Adv. Bioinformatics \\
Bioch 711 & Sequence Analysis \\
ChE 782 & Modeling Bio. Processes \\
ISyE 617 & Health Information Systems
\end{tabular}

\section{Biological Courses (3 required)}

Credit for lof the 3 required courses will be granted for: Intro. to Molecular Biology; Organic Chemistry, or Phys. Chemistry

\begin{tabular}{l|l}
\hline Gen 466 & General Genetics \\
Gen 612 & Prokaryotic Molecular Genetics \\
Bioch 501 & Intro. to Biochemistry \\
Bioch 601 & $\begin{array}{l}\text { Protein \& Enzyme Structure \& } \\
\text { Function } \\
\text { Cellular Signal Transduction Mech. }\end{array}$ \\
Bioch 630 & Macromolecular Crystallography \& \\
Bioch 636 & $\begin{array}{l}\text { Dynamics } \\
\text { Biophysical Chemistry }\end{array}$ \\
Bioch 665 & Bioch. Mechanisms of Cell. \\
Bioch 702 & Regulation \\
Gen 677 & Genomic Science \\
AHABS & Comp. Microbial Genomics \\
$375 / 875$ &
\end{tabular}
Genetics in eukaryotes and prokaryotes. Molecular basis of bacterial physiology and genetics with emphasis on molecular mechanisms. Chemistry, nutrition, and metabolism of biological systems.

Protein structure and dynamics. Protein folding. Physical organic chemistry of enzymatic catalysis. Analysis of enzyme kinetics and receptor-ligand interactions.

Descriptive statistics, distributions, one- and two-sample normal inference, power, one-way ANOVA, simple linear regression, categorical data, etc. expression data analysis, etc.

Advanced course covering computational problems in molecular biology and a core set of widely used algorithms.

Topics include genetic change; sequencing methods; comparison and alignment algorithms; motif and structure recognition; database searching. Course covers state-of-the-art tools to develop quantitative, predictive models of biological processes.

Covers core concepts of health information systems. Major applications include clinical information systems, language and standards, decision support, image technology and digital libraries.

Comprehensive coverage of human hormones, growth factors and other mediators; emphasis on hormone action and biosynthesis and hormoneproducing cells.

Obtaining detailed structural and dynamic information about biological macromolecules and survey results. X-ray diffraction, electron microscopy and molecular dynamics simulations.

Equilibrium thermodynamics, chemical kinetics and transport properties, with emphasis on solution behavior and application to noncovalent interactions of biological macromolecules in solution.

Control of major cellular metabolic pathways of biosynthesis and degradation; signal transduction; membrane biogenesis and cell compartmentation; intracellular traffic.

Provides knowledge of basics of genomic science and introduce students to cutting edge topics. Basics will be covered in both the physical and biological sciences.

Introduction to genomics with an emphasis on microbial model organisms and agents of infectious disease.

Computer Sciences Courses (3 required)

\begin{tabular}{|c|c|c|}
\hline CS 367 & Intro. to Data Structures & $\begin{array}{l}\text { Study of data structures (including stacks, queues, trees, graphs, and hash tables) and their applications. Analysis of efficient data structures and } \\
\text { algorithms. }\end{array}$ \\
\hline CS 514 & Numerical Analysis & $\begin{array}{l}\text { Polynomial forms, divided differences. Polynomial interpolation. Polynomial approximation: uniform approximation and Chebyshev polynomials, } \\
\text { least-squares approximation and orthogonal polynomials. Splines, B-splines, spline approximation. }\end{array}$ \\
\hline CS 525 & Linear Programming Methods & $\begin{array}{l}\text { Real linear algebra over polyhedral cones; theorems of the alternative for matrices. Formulation of linear programs. Duality theory \& solvability. The } \\
\text { simplex method \& related ones for efficient computer solution. Perturbation \& sensitivity analysis. }\end{array}$ \\
\hline CS 540 & Intro. to Artificial Intelligence & $\begin{array}{l}\text { Principles of knowledge-based search techniques; automatic deduction; knowledge representation using predicate logic, machine learning, semantic } \\
\text { networks, connectionist networks, etc. }\end{array}$ \\
\hline CS 559 & Computer Graphics & $\begin{array}{l}\text { Image representation, formation, presentation, composition and manipulation. Modeling, transformation, and display of geometric objects in two and } \\
\text { three dimensions. }\end{array}$ \\
\hline CS 564 & Database Managem't Systems & $\begin{array}{l}\text { Database management systems; different data models for structuring the logical view of the database: relational, hierarchical, and network. } \\
\text { Implementation techniques. }\end{array}$ \\
\hline CS 577 & Intro. to Algorithms & $\begin{array}{l}\text { Survey of important and useful algorithms for sorting, searching, pattern-matching, graph manipulation, geometry, and cryptography. Paradigms for } \\
\text { algorithm design. }\end{array}$ \\
\hline CS 635 & $\begin{array}{l}\text { Tools \& Environments for } \\
\text { Optimization }\end{array}$ & $\begin{array}{l}\text { Formulation and modeling of applications from computer sciences, operations research, business, science and engineering involving optimization and } \\
\text { equilibrium models. Survey and appropriate usage of software tools for solving such problems. }\end{array}$ \\
\hline \multicolumn{3}{|c|}{ and Informatics in Biology and } \\
\hline BMI 915 & CIBMS & $\begin{array}{l}\text { Weekly se } \\
\text { biomedica }\end{array}$ \\
\hline
\end{tabular}


weekly seminar during fall and spring semesters that brings trainees, trainers, and other interested faculty and students together for cross-disciplinary exposure to current research in computer science, biostatistics, engineering, biological sciences and biomedical research problems related to bioinformatics and computational biology. Presentations are designed to communicate across disciplines and are provided by CIBM trainees, UW-Madison faculty, and faculty from other institutions. The seminar is open to students, faculty, and others interested in bioinformatics. Another excellent training opportunity is the annual CIBM Program fall retreat, which features poster sessions where trainees as well as others in biomedical informatics share their research. Presentations include featured speakers by national and campus informatics scholars. Each year, one of the featured speakers is a UW bioinformatics alum.

\section{Research Training}

The driving forces for the research training opportunities arise from health and life sciences phenomenon. In many cases these questions demand new approaches and technologies. The weaving of cultures from computer sciences, biological sciences, and health sciences are illustrated in short descriptions of a sampling of the many wide-ranging multidisciplinary projects on campus (see Table 3 ). These

Table 3 Examples of Interdisciplinary Research with CIBM Faculty and Trainees

\begin{tabular}{|c|c|c|}
\hline Topic & ${ }^{1}$ Trainers, ${ }^{2}$ Trainees, $\&{ }^{3}$ Others & Focus \\
\hline $\begin{array}{l}\text { Whole-Genome Multiple } \\
\text { Alignment }\end{array}$ & $\begin{array}{l}\text { 'Perna } \\
{ }^{2} \text { Darling }\end{array}$ & $\begin{array}{l}\text { Computing whole-genome multiple alignments in the presence of large-scale evolutionary events. Developed algorithm } \\
\text { for multiple-genome alignment called Mauve (http://gel.ahabs.wisc.edu/mauve/). }\end{array}$ \\
\hline \multirow[t]{2}{*}{$\begin{array}{l}\text { Inducing Models of Regulatory } \\
\text { Networks }\end{array}$} & $\begin{array}{l}\text { 'Blattner, Craven, Page } \\
{ }^{2} \text { Bockhorst, Durfee, McFarlin, Noto, } \\
\text { Ong }\end{array}$ & $\begin{array}{l}\text { Developing novel machine-learning algorithms for uncovering gene-regulatory networks. Current and future research } \\
\text { directions include a) integrating regulatory-network with metabolic-network models, b) developing methods that are } \\
\text { able to exploit all relevant sources of data, including the scientific literature, and c) applying the methods to aid in the } \\
\text { understanding of disease-related data sets. }\end{array}$ \\
\hline & $\begin{array}{l}{ }^{1} \text { Craven, Kendziorski \& Yandell, } \\
{ }^{2} \text { Noto } \\
{ }^{3} \text { Attie (Biochemistry) }\end{array}$ & Model and understand large gene-expression, genotype and metabolomics data sets that pertain to type II diabetes. \\
\hline \multirow[t]{3}{*}{ Structural Bioinformatics } & $\begin{array}{l}\text { 'Phillips, Shavlik } \\
{ }^{2} \text { DiMaio, Soni }\end{array}$ & $\begin{array}{l}\text { Developing new algorithms for automatically interpreting electron density maps based on pictorial structures and } \\
\text { matching. Future directions include employing fully connected Bayesian networks, using machine learning to recognize } \\
\text { individual amino acids in electron density maps, scaling up to larger proteins, and handling poorer quality electronic } \\
\text { density maps. }\end{array}$ \\
\hline & $\begin{array}{l}{ }^{1} \text { Bannen, Kondrashov } \\
{ }^{2} \text { Cui, Phillips }\end{array}$ & $\begin{array}{l}\text { Developing simplified models to describe the equilibrium fluctuations near the native state, as opposed to non- } \\
\text { equilibrium processes, such as folding or induced-fit relaxation. }\end{array}$ \\
\hline & $\begin{array}{l}\text { 'Cunningham, Darnell, Marcia } \\
{ }^{2} \text { Mitchell }\end{array}$ & $\begin{array}{l}\text { Developing mathematical and computational methods for describing protein shape and for predicting the structure of } \\
\text { macromolecular complexes defined by two interacting proteins. Developing systems biology models for metal reduction } \\
\text { and carbon fixation pathways for bio-energy and bioremediation applications. }\end{array}$ \\
\hline $\begin{array}{l}\text { Simulations of Biomolecular } \\
\text { Information Processing }\end{array}$ & $\begin{array}{l}\text { IYin, } \\
{ }^{2} \text { Haseltine, Suthers } \\
{ }^{3} \text { Gourse (Bacteriology) }{ }^{3} \text { Rawlings } \\
\text { (Chemical \& Biological } \\
\text { Engineering) }\end{array}$ & $\begin{array}{l}\text { Write and solve mass-action kinetic models with a focus on simulating the intracellular/viral responses to nutrient } \\
\text { shifts. They have pioneered genome-to-organism dynamic models for diverse viruses. Fundamental advances include } \\
\text { identifying protein synthesis as the limiting resource for virus growth, quantifying how interactions among genes } \\
\text { contribute to virus fitness and robustness, and identifying conditions under which wild-type genome designs are } \\
\text { optimal. }\end{array}$ \\
\hline $\begin{array}{l}\text { Statistical-Relational Learning } \\
\text { Methods Applied to } \\
\text { Mammography }\end{array}$ & $\begin{array}{l}\text { 'Burnside, Page, Shavlik } \\
{ }^{2} \text { Davis }\end{array}$ & $\begin{array}{l}\text { Develop novel statistical relational learning (SRL) algorithms and apply them to the task of creating an expert system } \\
\text { for mammography. They have shown that SRL algorithms can benefit from the ability to define new data views that } \\
\text { can enhance the accuracy of predicting important fields in the original database. }\end{array}$ \\
\hline $\begin{array}{l}\text { Informatics for Clinical and } \\
\text { Operational Support }\end{array}$ & $\begin{array}{l}{ }^{1} \text { Brennan } \\
{ }^{2} \text { Haight, Hsieh, Severtson, Zayas- } \\
\text { Caban } \\
{ }^{3} \text { Karsh, Caryon, Robinson } \\
\text { (Industrial and Systems } \\
\text { Engineering) }\end{array}$ & $\begin{array}{l}\text { Research in the Brennan lab is aimed at supporting informed decisions at multiple levels within the healthcare system. } \\
\text { The HeartCare II project developed, deployed and is evaluating the impact of technology enhanced nursing practice in } \\
\text { home care settings. A recently established, Robert Wood Johnson-supported national coordinating center for personal } \\
\text { health records brings academic and industry partners together to solve the challenge of creating a seamless link across } \\
\text { all health information related to a given person. Mathematical modeling projects include employing Markov models for } \\
\text { optimal treatment sequencing decisions, quality engineering and forecasting models to improve access to care for } \\
\text { people who need treatment for substance abuse, stochastic simulation and real options models to the challenge of } \\
\text { pricing health information exchange alliances. }\end{array}$ \\
\hline
\end{tabular}

1 CIBM Faculty Trainers, 2 CIBM Trainees, 3 Other Faculty 
examples illustrate the CIBM culture of research that generates productive interdisciplinary research training.

A key focus of research at UW-Madison is the development of novel bioinformatics algorithms to analyze molecular data, genome sequences, proteins (levels, interactions, structures), and regulatory pathways. These advances are only possible when computer scientists understand enough about the problems to design usable tools and when bioscientists understand what is possible using computational and information technologies. The CIBM Program has established a culture of research that supports the development of these state-of-the-art bioinformatics algorithms. Trainees and faculty have published papers in the leading bioinformatics venues on such critical topics as multiple genome alignment [2], identification of regulatory structures in a genome [3], and analysis of high-throughput biological data [4] including expression data from microarrays [5], single-nucleotide polymorphism data [6], and structural bioinformatics [7 - 13].

As bioinformatics algorithms contribute more to our understanding of biology at a molecular level, there is a need for these algorithms to have a more direct impact on medical diagnostic and treatment processes. The CIBM Program recently added a unique translational medicine component through a collaboration with the Marshfield Clinic expanding its focus to span from molecular data to disease prediction and prevention.

An example project in the translational medicine component focuses on the design and deployment of machine learning algorithms to predict responses to a particular treatment from combined single-nucleotide polymorphism (SNP) data and clinical history data. This "Molecules to Bedside" component (see
Figure 1) complements CIBM's tradition of bioinformatics research and expands it - giving trainees the opportunity to apply their bioinformatics algorithms directly to clinical data. The Marshfield Clinic has an electronic medical record that dates back to the 1960s and a new personalized medicine program that already has collected DNA for nearly 20,000 of its patients for genotyping [14]. The clinic is the sole health care provider for many people in central Wisconsin including these 20,000. The Marshfield Clinic's database is a rare resource of detailed clinical and growing genetic data on patients [15].

\section{Informatics Training within UW-Madison Departments}

In addition to the CIBM Program, UWMadison students have multiple options for pursuing biomedical informatics training. Options include a degree minor, certificate programs, research training programs in various informatics areas, and courses that provide an informatics component within graduate programs. Many of the faculty that provide biomedical informatics training within these departments are CIBM faculty thus providing an integration between these training programs. Formal courses that provide the foundation for academic training in biomedical informatics are housed in various departments. We next provide an overview of the biomedical informatics components within these departments.

Department of Biostatistics and Medical Informatics. The Department of Biostatistics and Medical Informatics, within the School of Medicine and Public Health, serves as a resource for clinical, population and basic sciences investigators. A major goal of the Department faculty and staff is to collaborate in the design, conduct, and analyses of laboratory, clinical, and epidemiologic studies in a variety of biomedical disciplines and departments. Faculty conduct research in statistical methodology and computational methods and participate in three graduate and postdoctoral training programs - one of these is a program in medical informatics. Medical informatics training is offered via 1) a degree minor from this department that can be satisfied by courses in the CIBM Program's curriculum, 2) MS and $\mathrm{PhD}$ programs in Computer Sciences, 3) a Graduate Certificate program for students cur-

\begin{tabular}{|ll|}
\hline Bedside & Treatment Response \\
Disease Susceptibility \\
& Lab Results \\
& Symptoms \\
& Tissues \\
& Proteins \\
& mRNAs \\
& Genotype (SNPs, etc.) \\
\hline
\end{tabular}

Fig. 1 Molecules to Bedside. CIBM faculty and students are developing algorithms to predict items closer to the bedside based on items closer to the molecular level. For example, we may be able to predict disease susceptibility or response to treatment based on genotype or gene expression. CIBM trainees and faculty have published papers predicting symptoms from protein levels, predicting disease state from mRNAs, and predicting disease susceptibility from SNPs. 
rently enrolled in medical science or biological science graduate programs, and 4) a Capstone Certificate program for post-doctoral fellows and employees of local companies working in molecular biology.

Biomedical informatics research, offered in three main areas, illustrates how computation is integrated with medical applications in this department. Professors Craven, Kendziorski, Newton, Page, and Shavlik offer research projects in genetics/genomics. Projects include characterization of the genetic factors involved in susceptibility and resistance to cancer growth, mapping gene locations, analyzing genomic sequence data, analysis of mutagenesis experiments, analyzing gene-expression array data, optical mapping of genomes, predicting genomic regulatory elements, algorithms for analysis of mass spectrometry data, and novel gene-expression measurement technology. Collaborators include the UW Comprehensive Cancer Center, McArdle Laboratory for Cancer Research, Computer Sciences, Pharmacy, Genetics, and Biochemistry. Professors Craven, Page, Shavlik, Burnside and DeMets offer research projects in clinical informatics and bioinformatics. Collaborative projects include computational diagnosis of breast cancer including the determination of which mammogram abnormalities require biopsy, automated methods for extracting information from the biomedical literature, automated interpretation of images from $x$-ray crystallography, and automated pharmacophore discovery to help guide drug design. Collaborators include the UW Comprehensive Cancer Center, McArdle Laboratory for Cancer Research, Computer Sciences, Pharmacy, Genetics, Biochemistry, and the General Clinical Research Center.

Professors Chung and Dyer are developing algorithms for analyzing MRI images of the brain, where the algorithms perform segmentation, co-registration, and description. Collaborations exist with the Keck Lab (brain imaging), the Department of Ophthalmology and Visual Sciences, the Fundus Photograph Reading Center, Medical Physics, Mechanical Engineering, Waisman Center (speech kinematics), and the Department of Radiology (diagnostic and functional MRI).

Department of Computer Sciences. The Department of Computer Sciences is consistently ranked as one of the top ten computer science departments in the country. Most of UW's formal biomedical informatics courses are offered through this department. In particular, the department has internationally recognized research programs in Artificial Intelligence (primarily Computer Vision and Machine Learning), Computational Biology, Computer Architecture and VLSI (Very Large Scale Integration), Computer Graphics, Computer Networks, Computer Security, Database Systems, Numerical Analysis, Operating Systems, Optimization, Performance Analysis, Programming Languages and Compilers, and Theoretical Computer Science - all of which have relevance to biomedical informatics applications.

A recent project, relevant to both biomedical and public health science, is a collaboration between Professors DeWitt and Shavlik (Computer Science), Hanrahan (Chief Epidemiologist, State of Wisconsin), and Trentham-Dietz (Population Health Sciences) to simultaneously develop algorithms for anonymizing data along with machinelearning algorithms capable of extracting useful information from anonymized data. Maximizing the tradeoff between these two conflicting goals will allow significant scientific knowledge to be extracted from biomedical datasets while guarding patient privacy.
Health Systems. Clinical informatics training occurs in a unique environmental spanning the Department of Industrial and Systems Engineering and the School of Nursing. Professor Brennan teaches graduate level health informatics classes in the Department of Industrial and Systems Engineering and the School of Nursing. Training within these departments is primarily in the area of clinical informatics within the health care system. Curricula consist of established courses (for example, Health Systems Design) and special courses. For example, she recently partnered with faculty from three other universities that have a nursing informatics program to offer a cross campus course in Nursing Informatics. This endeavor was under the umbrella of the Committee on Institutional Cooperation (CIC) among ten leading Midwest universities known as the Big Ten. Her multidisciplinary research, summarized in Table 2, is aimed toward developing informatics solutions that support informed decisions across the health care spectrum - from personal health to RHIO (Regional Health Information Organization) networks. The Brennan Health Systems Lab, comprised of trainees in Industrial and Systems Engineering and Nursing, offers a unique mix of perspectives that bring engineering knowledge to patient care, and the human care dimension to engineering approaches that makes a distinctive contribution to the biomedical informatics literature [16-17]. A hallmark of training with the Brennan lab is that each trainee develops and executes his or her own research. In some cases it directly interfaces with funded lab research, in other cases it has conceptual relevance but is in a different area.

Dr. Brennan also provides informatics training that falls outside of a traditional curriculum. For example, trainees in the 
nursing program participate in a video conference-mediated bi-monthly CIC Nursing Informatics Journal Club with participants from Nursing Informatics Training programs at four of the Big Ten universities: Indiana University, University of Iowa, Michigan State University, and University of Minnesota. Participants take turns selecting the topic, literature, and leading the discussion. She is also providing training to UW-Madison and UW-System Clinical Nurse Instructors for incorporating informatics and genomics content into nursing curriculum. These non-traditional training initiatives provide a mechanism for building collegial relationships and scholarship among nursing informatics researchers, and for translating clinical genomics into nursing practice via a train-the-trainer approach.

Health systems informatics research is supported by several center programs that allow trainees to participate in informatics research with various health care applications. The Center for Productivity and Quality Improvement, housed in the Department of Industrial and Systems Engineering, applies human factors solutions to health information systems implementation. Current projects evaluate the effect of computerized-provider order entry systems on clinical outcomes and examine the impact of bar code technologies on safe medication administration. This group also benchmarks technology acceptance patterns among health care workers and patients. The Trace Research and Development Center develops and deploys adaptive technologies to insure that people with all levels of abilities are able to effectively use electronic health care resources. The Center for Health Systems Research and Analysis houses a national repository for quality assessment in long term care.

\section{Informatics Training via Individual Fellowships, Traineeships and Mentorships}

Biomedical informatics training at UWMadison is also supported via individual training fellowships. Two informatics fellows funded by the NLM are from the Brennan lab. In one exemplary program, a doctoral candidate at the School of Nursing constructed a secondary emphasis in nursing informatics through intra and extra-mural training. She has a minor in Computer Sciences and took five courses in this department that support her interest in developing computable representations that accurately characterize concepts relevant to nursing practice. Informatics expertise that is represented on her dissertation committee include Dr. Brennan and faculty members from the UW-Madison School of Library and Information Studies as well as Stanford Medical Informatics. She participated in genomics training offered by the Jackson Laboratory in Bar Harbor and works with Dr. Brennan and Clinical Faculty at UW-Madison to incorporate informatics and genomics into the undergraduate nursing curriculum. An NLM-funded postdoctoral fellow works with Brennan's HeartCare II research team. She is applying Brennan's model of technology enhanced nursing practice toward developing informatics resources to support a model of integrated care for adolescents in the area of mental health. A NLM-funded career fellow in the Department of Biochemistry, and former CIBM postdoctoral fellow, is conducting research that will accelerate understanding proteins and their structures through the development of tools aimed to significantly speed up understanding of protein structures by building a probabilistic framework that integrates informatics and physical models. These examples show how biomedical informatics training can occur outside of a structured informatics program and can meet training needs over the course of one's career.

\section{Conclusions}

Biomedical informatics training at UWMadison is provided across a range of disciplines and is available at different training stages. The CIBM program illustrates how a cross-training program can support highly productive biomedical informatics research training outside of a degree granting biomedical informatics department. This program fosters a creative synergy that advances biomedical informatics across multiple fronts and prepares trainees to participate in an evolving and multidisciplinary field. Biomedical informatics training offered within established departments adheres to a similar model of immersing students in multidisciplinary training and education that is supported by faculty trainers that are conducting collaborative research across departments. The health systems informatics training complements the bioscience focus of the other programs and fosters the application of biomedical informatics to health care. Individual fellowships support the development of biomedical informatics expertise through individualized training programs. UW-Madison, with multiple departments across biological, computational and health fields, provides a rich biomedical informatics training environment that has been harnessed to create productive biomedical training programs.

\section{Acknowledgements}

We thank the NLM for its support of the CIBM Training Program (Grant No. 
Severtson et al.

T15LM007359). Additional support to CIBM comes from the Genome Center of Wisconsin, the Department of Biochemistry, the Department of Biostatistics and Medical Informatics, and the UW-Madison Graduate School.

\section{References}

1. Friedman CP, Altman RB, Kohane IS, McCormick KA, Miller PL, Ozbolt JG, et al. Training the next generation of informaticians: the impact of "BISTI" and bioinformatics-A Report from the American College of Medical Informatics. J Am Med Inform Assoc 2004 Jan 11:167-72.

2. Darling A, Mau B, Blattner F, Perna N. Mauve: Multiple alignment of conserved genomic sequence with rearrangements. Genome Res $2004 \mathrm{Jul}$ 14(7):1394-403.

3. Bockhorst J, Qiu Y, Glasner J, Liu, M, Blattner F Craven M. Predicting bacterial transcription units using sequence and expression data. Bioinformatics (special issue: Proceedings of the 11th International Conference on Intelligent Systems for Molecular Biology), 200319 Suppl 1:34i-43i.

4. Molla M, Waddell M, Page D, Shavlik J. Using machine learning to design and interpret geneexpression microarrays. AI Magazine 2004; 25:23-44.

5. Ong J, Glasner J, Page D. Modeling regulatory pathways in E. coli from time series expression profiles. Published in Bioinformatics supplement as Proceedings of the International Conference on Intelligent Systems for Molecular Biology (ISMB02) 2002. p. 241-8.

6. Waddell M, Page D, Zhan F, Barlogie B, Shaughnessy J. Predicting cancer susceptibility from single-nucleotide polymorphism data: a case study in multiple myeloma. Appears in the Proceedings of the 5th international workshop on Bioinformatics, Conference on Knowledge Discovery in Data 2005 p. 21-8.

7. DiMaio F, Shavlik J, Phillips GN. Pictorial structures for molecular modeling. Adv Neural Inf Process Syst (NIPS). MIT Press 2005;17:369-76.

8. DiMaio F, Shavlik J, Phillips GN. A probabilistic approach to protein backbone tracing in electron density maps. Bioinformatics 2006 Jul 15;22(14):e81-9.

9. Han BW, Bingman CA, Mahnke DK, Bannen RM, Bednarek S, Sabina RL, et al. Membrane association, mechanism of action and structure of Arabidopsis embryonic factor 1 (FAC1). J Biol Chem 2006 May 26;281(21):14939-47.

10. Kondrashov DA and Phillips GN Jr. Molecular mastication mechanics. Structure 2005 Jun; 13:836-7.

11. Kondrashov DA, Cui Q, Phillips GN Jr. Optimization and evaluation of a coarse-grained model of protein motion using $\mathrm{x}$-ray crystal data. Biophys J 2006 Oct 15;91(8):2760-7. Epub 2006 Aug 4.

12. Kondrashov DA, Van Wynsberghe AW, Bannen RM, Cui Q, Phillips GN Jr. Protein structural variation in computational models and crystallographic data. Structure 2007 Feb;15(2):169-77.
13. Eghbalnia HR, Wang L, Bahrami A, Assadi A, Markley JL. Protein energetic conformational analysis from NMR chemical shifts (PECAN) and its use in determining secondary structural elements. J Biomol NMR 2005 May;32(1):71-81.

14. McCarty CA, Wilke RA, Giampietro PF, Wesbrook SD, Caldwell MD. Marshfield Clinic Pers \}onalized Medicine Research Project (PMRP): design, methods and recruitment for a large population-based biobank. Personalized Medicine 2005;2(1):49-79.

15. Greenlee RG. Measuring disease frequency in the Marshfield epidemiologic study area (MESA). Clin Med Res 2003;1(4):273-80.

16. Kossman S, Casper GR, Severtson DJ, Grenier,AS, Or C, Carayon P, et al. Designing study nurses' training to enhance research integrity: A macroergonomic approach. Proceedings of the $9^{\text {th }}$ Annual meeting of the American Medical Informatics Association. Washington DC. November 10 - 14, 2006. p. 439-43.

17. Carayon P, Schoofs Hundt A, Karsh B-T, Gurses AP, Alvarado CJ, Smith M, et al. Work system design for patient safety: The SEIPS Model. Qual Saf Health Care 2006; 15(Suppl 1): i50-i58.

\section{Correspondence to:}

Dolores J. Severtson

H6/295 CSC

600 Highland Ave.

Madison, WI 53575, USA

Tel: +1 608-263-5311

Fax: +1 08-263-5332

E-mail: disevert@wisc.edu 